\title{
Obesity, chronic disease, age, and in- hospital mortality in patients with covid-19: analysis of ISARIC clinical characterisation protocol UK cohort
}

Thomas Yates ${ }^{1,2^{*}}$, Francesco Zaccardi ${ }^{1,3}$, Nazrul Islam ${ }^{4,5}$, Cameron Razieh ${ }^{1,2}$, Clare L. Gillies ${ }^{1,3}$, Claire A. Lawson ${ }^{1}$, Yogini Chudasama ${ }^{3}$, Alex Rowlands ${ }^{1,2}$, Melanie J. Davies ${ }^{1,2}$, Annemarie B. Docherty ${ }^{6,7}$, Peter J. M. Openshaw ${ }^{8}$, J. Kenneth Baillie ${ }^{9}$, Malcolm G. Semple ${ }^{10,11}$ and Kamlesh Khunti ${ }^{1,3,12}$

\begin{abstract}
Background: Although age, obesity and pre-existing chronic diseases are established risk factors for COVID-19 outcomes, their interactions have not been well researched.

Methods: We used data from the Clinical Characterisation Protocol UK (CCP-UK) for Severe Emerging Infection developed by the International Severe Acute Respiratory and emerging Infections Consortium (ISARIC). Patients admitted to hospital with COVID-19 from 6th February to 12th October 2020 were included where there was a coded outcome following hospital admission. Obesity was determined by an assessment from a clinician and chronic disease by medical records. Chronic diseases included: chronic cardiac disease, hypertension, chronic kidney disease, chronic pulmonary disease, diabetes and cancer. Mutually exclusive categories of obesity, with or without chronic disease, were created. Associations with in-hospital mortality were examined across sex and age categories.

Results: The analysis included 27,624 women with 6407 (23.2\%) in-hospital deaths and 35,065 men with 10,001 (28.5\%) in-hospital deaths. The prevalence of chronic disease in women and men was 66.3 and 68.5\%, respectively, while that of obesity was 12.9 and $11.1 \%$, respectively. Association of obesity and chronic disease status varied by age $(p<0.001)$. Under 50 years of age, obesity and chronic disease were associated with in-hospital mortality within 28 days of admission in a dose-response manner, such that patients with both obesity and chronic disease had the highest risk with a hazard ratio (HR) of in-hospital mortality of 2.99 (95\% Cl: $2.12,4.21$ ) in men and $2.16(1.42,3.26)$ in women compared to patients without obesity or chronic disease. Between the ages of 50-69 years, obesity and chronic disease remained associated with in-hospital COVID-19 mortality, but survival in those with obesity was similar to those with and without prevalent chronic disease. Beyond the age of 70 years in men and 80 years in women there was no meaningful difference between those with and without obesity and/or chronic disease.
\end{abstract}

\footnotetext{
* Correspondence: ty20@leicester.ac.uk; ty20@le.ac.uk

'Diabetes Research Centre, Leicester General Hospital, University of Leicester, Leicester LE5 4PW, UK

${ }^{2}$ National Institute for Health Research (NIHR) Leicester Biomedical Research Centre (BRC), Leicester General Hospital, Leicester LE5 4PW, UK

Full list of author information is available at the end of the article
}

(c) The Author(s). 2021 Open Access This article is licensed under a Creative Commons Attribution 4.0 International License, which permits use, sharing, adaptation, distribution and reproduction in any medium or format, as long as you give appropriate credit to the original author(s) and the source, provide a link to the Creative Commons licence, and indicate if changes were made. The images or other third party material in this article are included in the article's Creative Commons licence, unless indicated otherwise in a credit line to the material. If material is not included in the article's Creative Commons licence and your intended use is not permitted by statutory regulation or exceeds the permitted use, you will need to obtain permission directly from the copyright holder. To view a copy of this licence, visit http://creativecommons.org/licenses/by/4.0/ The Creative Commons Public Domain Dedication waiver (http://creativecommons.org/publicdomain/zero/1.0/) applies to the data made available in this article, unless otherwise stated in a credit line to the data. 
Conclusion: Obesity and chronic disease are important risk factors for in-hospital mortality in younger age groups, with the combination of chronic disease and obesity being particularly important in those under 50 years of age. These findings have implications for targeted public health interventions, vaccination strategies and in-hospital clinical decision making.

Keywords: Obesity, Chronic disease, Ageing, COVID-19

\section{Introduction}

The severe acute respiratory syndrome coronavirus 2 (SARS-CoV-2), which causes coronavirus disease-2019 (COVID-19), is devastating global economies and putting unprecedented strain on health care services. The mortality and economic burden caused by the virus have precipitated an unparalleled global research response [1], including efforts to identify people at greatest risk of developing severe illness or mortality. Age, sex, chronic disease and obesity have emerged as key risk factors within large population level cohorts [2, 3]. Overweight and obesity have been suggested to increase the risk of severe disease or mortality by a factor of two [4], with diabetes, hypertension, coronary heart disease, chronic kidney disease, respiratory disease, and cancer all being shown to be associated with the risk of adverse COVID19 outcomes [5-8]. However, whilst these factors have been extensively investigated individually, their interactions are less well understood. It is unknown, for example, whether obesity remains a strong risk factor in those without chronic disease, or whether the combination of obesity with chronic disease increases the risk, given their common coexistence. Whilst studies investigating the associations of obesity and chronic disease with COVID-19 outcomes have been adjusted for age, sex or both, adjustment can render invisible heterogeneity in associations across strata of interest which can mask important differences, including within COVID19 research [9]. Early preliminary research in small cohorts has started to demonstrate the presence of heterogeneity for age, with obesity found to be more prevalent or a stronger risk factor for COVID-19 outcomes in younger compared to older populations [10-14], suggesting that younger people with obesity may be a priority group for public health testing and preventative strategies. However, not all research has supported these findings [15] and it is unknown whether age modifies associations between obesity and COVID-19 mortality equally in men and women or how obesity combines with associations of chronic disease. Examining how age modifies associations of combinations of common risk factors with COVID-19 outcomes will allow for more nuance and personalisation in risk communication, public health strategies and clinical decision making.
We use the ISARIC CCP-UK database to investigate associations between obesity, chronic disease and their combination with in-hospital death in patients admitted with COVID-19 and whether these associations are modified by age in men and women.

\section{Methods \\ Study population}

This study uses data from the Clinical Characterisation Protocol UK (CCP-UK) for Severe Emerging Infection developed by the International Severe Acute Respiratory and emerging Infections Consortium (ISARIC) and the World Health Organisation in response to the A/ H1N1pdm2009 influenza pandemic [16]. ISARIC CCPUK was reactivated in the UK on 17th January 2020 as the scale of the COVID-19 pandemic started to emerge. Data was collected from 260 hospitals in England, Scotland, and Wales. The protocol, amendment history, case report form, information leaflets, consent forms, and detail of the Independent Data and Material Access Committee (IDAMAC) are available at https://isaric4c. net. The study was approved by the South Central - Oxford C Research Ethics Committee in England (Ref: 13/ SC/0149), and by the Scotland Research Ethics Committee (Ref: 20/SS/0028). For this study, we included those with a coding of "Proven or high likelihood of infection with a pathogen of Public Health Interest" reflecting that a preparedness protocol cannot assume a diagnostic test will be available for an emergent pathogen. Site training also emphasised importance of only recruiting proven cases of COVID-19. Additional inclusion criteria were: a hospital admittance date from the start of the COVID19 pandemic in the UK (6th February 2020) with a complete follow-up coding indicating discharge, inhospital death, or remaining in hospital. We excluded patients coded as having had a solid organ transplant or people with rare diseases and inborn errors of metabolism that significantly increase the risk of infections (such as severe combined immunodeficiency (SCID) or homozygous sickle cell). Data were available up until 12th October 2020. Of the 76,327 individuals within this dataset, 62,689 (82\%) had a valid censoring date with complete data for age, sex, obesity and chronic disease (diabetes, chronic heart disease, hypertension, chronic kidney 
disease, chronic pulmonary disease or cancer) and were included in this analysis.

\section{Data collection}

Data collection was undertaken by research nurses, administrators and medical students. Detailed admission data were collected on day 1 with follow-up data on disease progression collected on day 3,6 , and 9, and on discharge, death or continued hospitalisation beyond 28 days. Discharge was further coded as discharged alive, discharged to palliative care, or discharged to another facility.

Obesity was coded as yes/no on assessment from the attending clinician. Clinical assessment was based on objective measurement of obesity, such as by calculation of the body mass index ( $\geq$ BMI of $30 \mathrm{~kg} / \mathrm{m}^{2}$ ), measurement of abdominal girth or on clinical judgment.

Chronic disease was based on clinician diagnosed status. In this study, we included diseases that have been consistently associated with COVID-19 outcomes [5-8]: chronic cardiac disease (coronary artery disease, heart failure, congenital heart disease, cardiomyopathy, rheumatic heart disease), hypertension, chronic kidney disease (diagnosed chronic kidney disease or estimated glomerular filtration rate $<60 \mathrm{~mL} / \mathrm{min} / 1.73 \mathrm{~m}^{2}$ ), chronic pulmonary disease (chronic obstructive pulmonary disease [chronic bronchitis, emphysema], cystic fibrosis, bronchiectasis, interstitial lung disease, pre-existing requirement for long term oxygen therapy), diabetes (type 1 or 2), and malignant neoplasm (current solid organ or haematological malignancy).

\section{Outcome}

The main outcome was in-hospital mortality coded as "death" for the patient outcome following hospital admittance. In order to avoid those with prolonged hospital stay biasing results, outcomes were censored at 28days.

\section{Statistical analysis}

The main analysis used a stratified rather than an adjusted model to investigate heterogeneity across strata. Data were stratified by age $(<50$ years, $50-59$ years, 60 69 years, $70-79$ years, $\geq 80$ years) and sex. Obesity and chronic disease were categorised into four mutually exclusive categories of interest: 1) non-obese without chronic disease, 2) obese without chronic disease, 3) non-obese with chronic disease, 4) obese with chronic disease. The Kaplan-Meier survival function was used to plot survival and generate survival tables. The log-rank test was used to determine overall differences in survival rates between obesity and chronic disease categories. Cox proportional hazards models were further used to quantify the relative hazard by age and sex strata for each combination of obesity and chronic disease compared to patients with no obesity and no chronic disease. In order to formally test whether age modified associations, an interaction test for age category by obesity/ chronic disease category was also analysed, separately in men and women.

Two sensitivity analyses were conducted. The first considered the potential for discharge to palliative care biasing results, particularly in older individuals, by repeating analysis for a composite outcome of in-hospital mortality or discharge to palliative care. The second sensitivity analysis included obesity and each chronic disease separately to confirm whether the overall pattern of association observed in the main analysis was consistent for each individual disease and obesity. All analysis was conducted in SPSS (v26). $P<0.05$ was used to denote significance for main effects and interactions. Data are reported as mean $(95 \% \mathrm{CI})$, unless reported otherwise.

\section{Results}

Table 1 shows the age, chronic disease and obesity characteristics of the included cohort. The analysis included 27,624 women and 35,065 men, with 6407 (23.2\%) and 10,001 (28.5\%) in-hospital deaths, respectively. The median number of days until discharge or death was between 8 and 9 days for both men and women. The proportion of patients with at least one chronic disease was 66.3 and $68.5 \%$ in women and men, respectively, while the prevalence of obesity was 12.9 and $11.1 \%$, respectively. Rates of chronic disease and obesity by age group are shown in Supplementary Table S1, with rates of chronic disease highest in those over 80 years of age $(83.6 \%)$ and rates of obesity highest in those $50-59$ years of age (20.5\%) and lowest in those over 80 years of age (4.7\%).

Figure 1 for men and Fig. 2 for women show the 28day survival across obesity, chronic disease and age categories, with Fig. 3 showing the accompanying hazard ratios (data shown in Supplementary Table S2). Associations between obesity and chronic disease categories with in-hospital mortality varied by age in men and women $(P<0.001$ for interaction).

For patients under 50 years of age, obesity and chronic diseases were associated with in-hospital mortality in a dose-response manner, such that those with both obesity and chronic disease had the lowest survival (Figs. 1 and 2), with a HR of 2.99 (95\% CI: 2.12, 4.21) in men and $2.16(1.42,3.26)$ in women compared to patients without obesity or chronic diseases (Fig. 3). In the age categories of 50-59 and 60-69 years, obesity and chronic disease remained associated with in-hospital COVID-19 mortality, but survival in those with obesity tended to be similar in those with and without coexisting chronic disease (Fig. 3). For example, in women between 50 and 59 
Table 1 Participant characteristics stratified by sex

\begin{tabular}{|c|c|c|c|c|c|c|}
\hline \multirow[t]{2}{*}{ Categorical Variables } & \multicolumn{3}{|c|}{ Men $(n=35,065)$} & \multicolumn{3}{|c|}{ Women $(27,624)$} \\
\hline & Number & Column \% & & Number & Column \% & \\
\hline \multicolumn{7}{|l|}{ Age Category } \\
\hline$<50$ years & 5398 & 15.4 & & 4586 & 16.6 & \\
\hline 50-59 years & 4408 & 12.6 & & 2745 & 9.9 & \\
\hline $60-69$ years & 5789 & 16.5 & & 3587 & 13.0 & \\
\hline 70-79 years & 8187 & 23.3 & & 5581 & 20.2 & \\
\hline 80 years & 11,283 & 32.2 & & 11,125 & 40.3 & \\
\hline Hypertension & 9756 & 46.4 & & 8234 & 46.3 & \\
\hline Chronic Heart Disease & 11,491 & 33.2 & & 7651 & 28.1 & \\
\hline Diabetes & 8379 & 24.8 & & 5364 & 20.1 & \\
\hline Chronic pulmonary disease & 5985 & 17.3 & & 4449 & 16.3 & \\
\hline Chronic Kidney Disease & 5553 & 16.0 & & 4583 & 16.8 & \\
\hline Cancer & 4197 & 12.1 & & 3161 & 11.6 & \\
\hline Any chronic disease & 24,007 & 68.5 & & 18,316 & 66.3 & \\
\hline Obesity & 3901 & 11.1 & & 3561 & 12.9 & \\
\hline \multicolumn{7}{|c|}{ Mutually exclusive categories of obesity and chronic disease } \\
\hline Non obese, no chronic disease & 10,103 & 28.8 & & 8259 & 29.9 & \\
\hline Obese, no chronic disease & 955 & 2.7 & & 1049 & 3.8 & \\
\hline Non-obese, with chronic disease & 21,061 & 60.1 & & 15,804 & 57.2 & \\
\hline Obese with chronic disease & 2946 & 8.4 & & 2512 & 9.1 & \\
\hline In-hospital mortality within 28 days & 10,001 & 28.5 & & 6407 & 23.2 & \\
\hline Discharged within 28 days & 20,388 & 58.1 & & 17,625 & 63.8 & \\
\hline Remain in hospital after 28 days & 4676 & 13.3 & & 3592 & 13.0 & \\
\hline Continuous Variables & Median & 25th percentile & 75th percentile & Median & 25th percentile & 75th percentile \\
\hline Days in hospital until death & 9 & 4 & 16 & 8 & 4 & 16 \\
\hline Days in hospital until discharge & 8 & 4 & 16 & 8 & 4 & 16 \\
\hline
\end{tabular}

years, compared to those without obesity or chronic disease, the HR of in-hospital mortality in those with obesity without chronic disease was $2.61(1.78,3.82)$ while the $\mathrm{HR}$ in those with obesity and chronic disease was $2.12(1.52,2.93)$. Between 70 and 79 years, there was no clear pattern of association between obesity and chronic disease in men, whereas risk in those with obesity and chronic disease remained moderately elevated in women $(\mathrm{HR}=1.33 ; 1.10,1.60)$ (Fig. 3). Beyond 80 years of age, survival was low across all categories of obesity and chronic disease status (Figs. 1 and 2) with no meaningful difference in survival between those without obesity and chronic disease compared to those with obesity and/or chronic disease in men and women.

The strength and pattern of association was unchanged when those discharged to palliative care $(\mathrm{men}=$ 437 , women $=459$ ) were included in a composite outcome with mortality (Supplementary Figure S1). The pattern of association with in-hospital mortality was also consistent across categories of age when obesity and each included chronic disease were analysed individually (Supplementary Figure S2), with associations weakest in older adults.

\section{Discussion}

In patients admitted to hospital with COVID-19, mutually exclusive categories of obesity and chronic disease are consistently associated with in-hospital mortality in younger adults but not in those 70 years of age or over for men or 80 years and over for women. In men and women under 50 years of age, obesity and the coexistence of chronic diseases combined to increase the risk of in-hospital mortality by between 2 to 3 times compared to those without obesity or chronic diseases. Between the ages of 50 to 69 years, obesity and chronic diseases remained associated with a higher risk of in-hospital mortality in men and women, but their combination did not appear to further reduce survival beyond having 

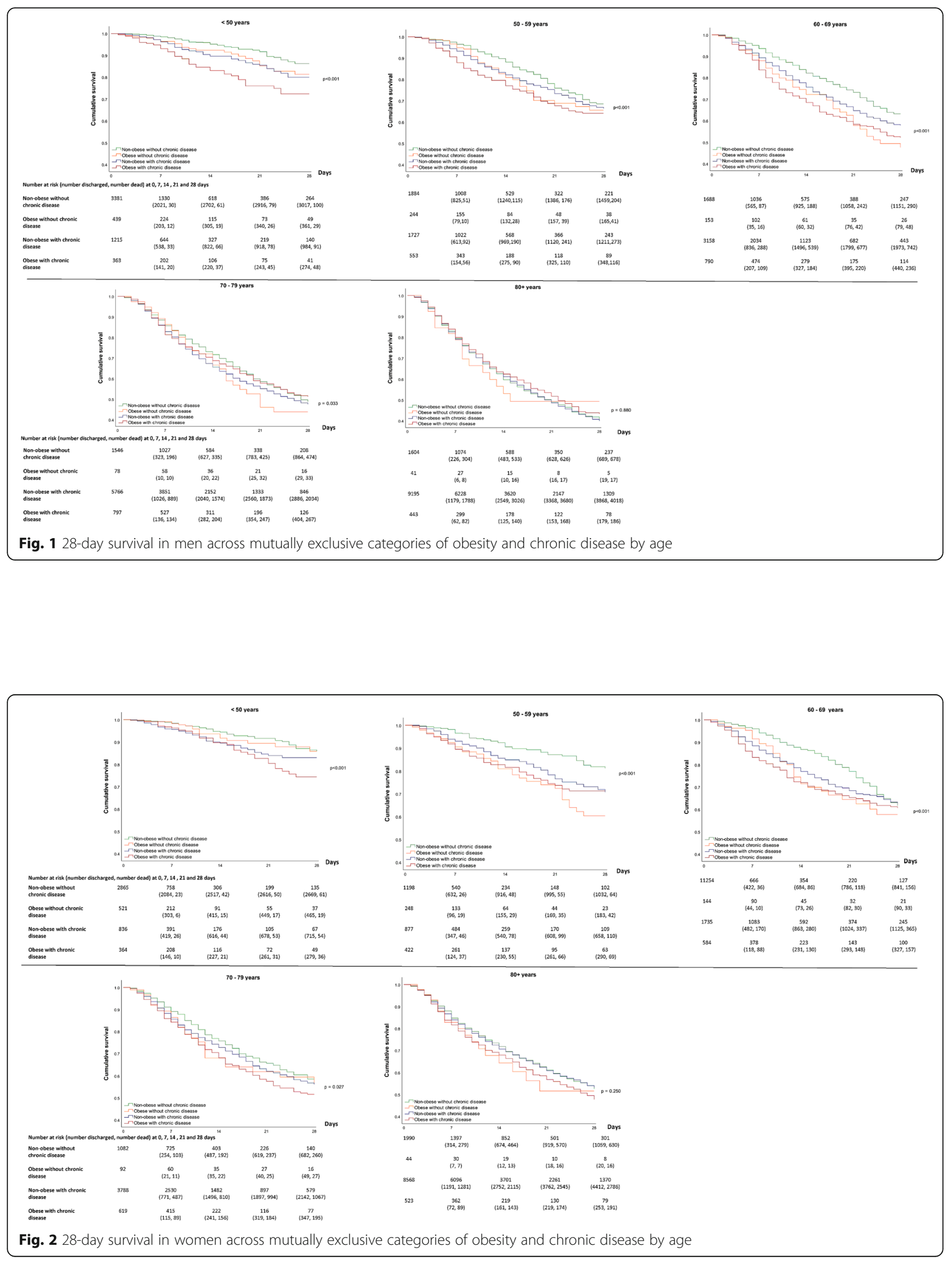


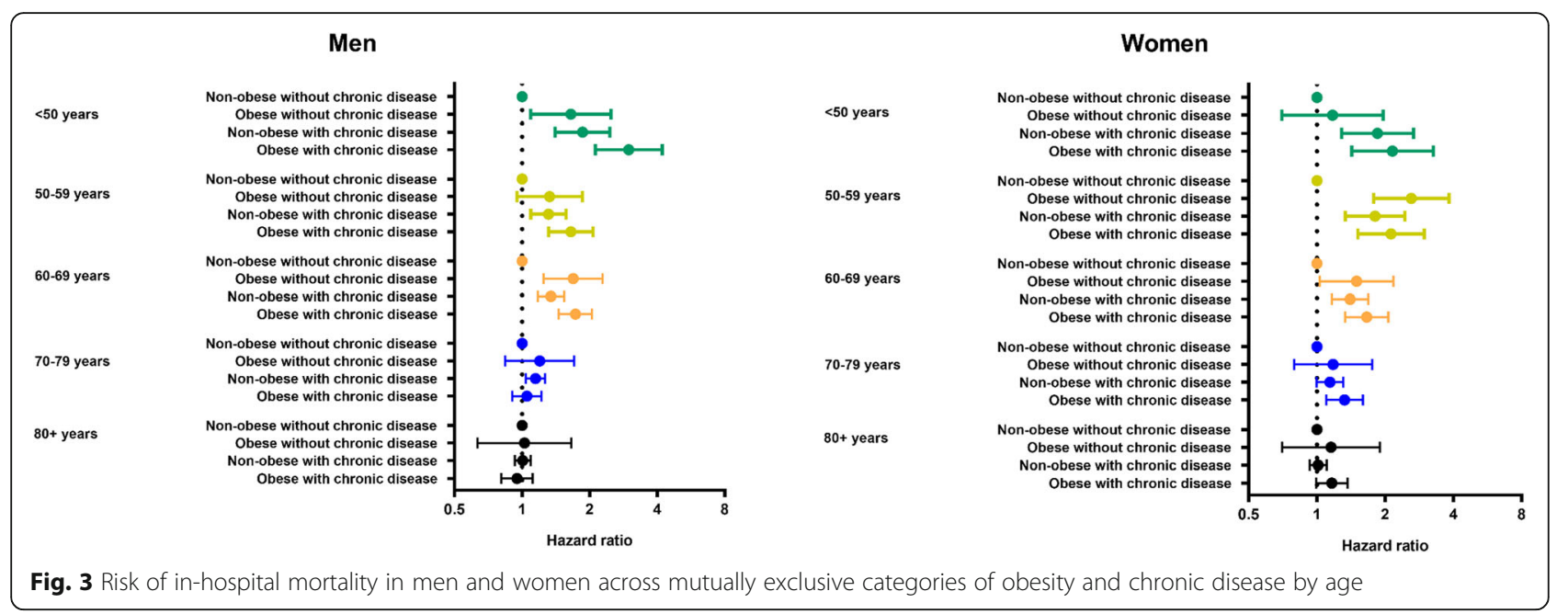

obesity only. In those aged 70 years or over in men and 80 or over years in women, survival was low across all combinations of obesity and chronic disease, but was not meaningfully further lowered in those with obesity and/or chronic disease.

Whilst obesity and chronic disease have been established as risk factors for SARS-CoV-2 infection, severe disease and mortality [2-8], there has been less focus on establishing how these risk factors combine in men and women and whether the pattern of association is consistent across different age groups. This study expands preliminary findings from smaller cohorts suggesting obesity may be a particularly important risk factor in younger ages. Among 7606 patients in the United States, associations of BMI with in-hospital mortality and mechanical ventilation were strongest in those under 50 years and largely attenuated in those over 70 years [14], which was consistent with an early study from a New York hospital system [10]. Another study from the USA found that in 6916 persons testing positive for COVID19 , obesity was more strongly associated with mortality in younger age men [12], while a study from Spain involving 1105 persons hospitalised with COVID-19 also reported that obesity was more strongly associated with admission in younger adults [13]. However, a study involving 5795 persons admitted to hospitals in Paris with COVID-19 suggested obesity was similarly associated with mortality across age groups [15], although uncertainty around the estimates of risk were high due to a low number of events when stratified by age. The present study suggests that, with cases of COVID-19 that are severe enough to warrant admission to hospital, the coexistence of obesity and chronic disease substantially reduces survival in those under 50 years of age, with obesity remaining a risk factor with or without the coexistence of chronic disease up until the age of 70 years. However, beyond this age, obesity with or without chronic disease may not meaningfully affect the already low levels of survival associated with older age, particularly in men. These findings broadly support the vaccination strategy within the United Kingdom [17], the first country to approve the roll-out of a COVID-19 vaccine. The strategy sets out health care workers and older adults (with priority set in descending age order until $\geq 65$ years) as the first priority, followed by those over 16 years of age with an underlying health condition, including morbid obesity and the chronic diseases included in this paper.

Strengths of this analysis include the large multisite sample with data collected from trained staff according to standard operating procedures. In the United Kingdom (UK), there were a total of 151,641 hospital admissions with COVID-19 up until October 12th (https:// coronavirus.data.gov.uk/). Therefore, our cohort represents $41 \%$ of all UK hospital admissions in the period covered by this study. This large sample size allowed for stratification by age, sex and mutually exclusive categories of obesity and chronic diseases, allowing survival estimates for each strata rather than relying on adjusted estimates which can mask important differences between groups. However, there are some limitations. Namely, the large sample size was only made possible by relying on clinical records: for example, obesity was defined by a clinician assessment. Therefore, the prevalence of obesity appears an under-estimate compared to levels that would have been expected based on population level estimates [18]. It is likely the coding of obesity in this study therefore reflects more extreme phenotypes of obesity likely to prompt a clinical coding. Other factors like age may also influence whether patients were assessed for obesity, which could act to bias the reported associations. Nevertheless, in order to inform clinical 
care, analysed risk factors need to reflect data that is readily available to treating clinical staff through clinical records. Therefore, the coding of obesity in this study may have real world utility as it corresponds to data coded within routine clinical care. Another potential limitation is that this study is specific to in-hospital mortality and does not include COVID-19 mortality events in the community. Post-acute COVID-19 discharge from hospital of patients into palliative care is increasingly recognised with 'long-COVID-19' [19], which may have acted to bias findings through longer-term hospital admissions followed by a high mortality risk once discharged. Nevertheless, associations were unchanged when patients discharged into palliative care were included within the outcome as a composite with mortality.

In conclusion, this study suggests obesity and chronic diseases act as important risk factors for lower in-hospital survival in younger age groups, with the combination of chronic disease and obesity being a particularly important risk factor in patients under 50 years of age. Associations appeared to be attenuated with age, such that obesity and chronic diseases had little impact on survival in men beyond 70 years and women beyond 80 years. These findings suggests that younger adults with obesity and pre-existing chronic disease may need to be targeted by public health interventions to reduce the risk of transmission or to engage with vaccination programmes. However, obesity and chronic disease may be less important or informative for clinical decision making in the treatment of COVID-19 for older adults, where other factors may be more relevant.

\section{Supplementary Information}

The online version contains supplementary material available at https://doi. org/10.1186/s12879-021-06466-0.

Additional file 1: Supplementary Table S1. Rates of obesity and chronic disease by age group. Supplementary Table S2. Hazard ratio estimates used to draw Fig. 3. Supplementary Figure S1. Hazard ratios for each strata when those discharged into palliative care were included with in-hospital mortality as a composite outcome. Supplementary Figure S2. Hazard ratios for each disease and obesity analysed individually.

\section{Acknowledgements}

The study protocol is available at https://isaric4c.net/protocols; study registry https://www.isrctn.com/ISRCTN66726260. This work uses data provided by patients and collected by the NHS as part of their care and support \#DataSavesLives. We are grateful to the 2648 frontline NHS clinical and research staff and volunteer medical students who collected the data in challenging circumstances; and the generosity of the participants and their families for their individual contributions in these difficult times. We also acknowledge the support of Jeremy J Farrar, and Nahoko Shindo.

ISARIC Coronavirus Clinical Characterisation Consortium (ISARIC4C) Investigators: consortium lead investigator: J Kenneth Baillie; chief investigator: Malcolm G Semple; co-lead investigator: Peter JM Openshaw; ISARIC clinical coordinator: Gail Carson; co-investigators: Beatrice Alex, Benjamin Bach, Wendy S Barclay, Debby Bogaert, Meera Chand, Graham S Cooke, Annemarie B Docherty, Jake Dunning, Ana da Silva Filipe, Tom Fletcher, Christopher A Green, Ewen M Harrison, Julian A Hiscox, Antonia Ying Wai Ho, Peter W Horby, Samreen ljaz, Saye Khoo, Paul Klenerman, Andrew Law, Wei Shen Lim, Alexander J Mentzer, Laura Merson, Alison M Meynert, Mahdad
Noursadeghi, Shona C Moore, Massimo Palmarini, William A Paxton, Georgios Pollakis, Nicholas Price, Andrew Rambaut, David L Robertson, Clark D Russell, Vanessa Sancho-Shimizu, Janet T Scott, Louise Sigfrid, Tom Solomon, Shiranee Sriskandan, David Stuart, Charlotte Summers, Richard S Tedder, Emma C Thomson, Ryan S Thwaites, Lance CW Turtle, Maria Zambon; project managers: Hayley Hardwick, Chloe Donohue, Jane Ewins, Wilna Oosthuyzen, Fiona Griffiths; data analysts: Lisa Norman, Riinu Pius, Tom M Drake, Cameron J Fairfield, Stephen Knight, Kenneth A Mclean, Derek Murphy, Catherine A Shaw; data and information system manager: Jo Dalton, Michelle Girvan, Egle Saviciute, Stephanie Roberts, Janet Harrison, Laura Marsh, Marie Connor, Sophie Halpin, Clare Jackson, Carrol Gamble; data integration and presentation: Gary Leeming, Andrew Law, Ross Hendry, James Scott-Brown; material management: William Greenhalf, Victoria Shaw, Sarah McDonald; outbreak laboratory volunteers: Katie A Ahmed, Jane A Armstrong, Milton Ashworth, Innocent G Asiimwe, Siddharth Bakshi, Samantha L Barlow, Laura Booth, Benjamin Brennan, Katie Bullock, Benjamin WA Catterall, Jordan J Clark, Emily A Clarke, Sarah Cole, Louise Cooper, Helen Cox, Christopher Davis, Oslem Dincarslan, Chris Dunn, Philip Dyer, Angela Elliott, Anthony Evans, Lewis WS Fisher, Terry Foster, Isabel Garcia-Dorival, William Greenhalf, Philip Gunning, Catherine Hartley, Antonia Ho, Rebecca L Jensen, Christopher B Jones, Trevor R Jones, Shadia Khandaker, Katharine King, Robyn T Kiy, Chrysa Koukorava, Annette Lake, Suzannah Lant, Diane Latawiec, L Lavelle-Langham, Daniella Lefteri, Lauren Lett, Lucia A Livoti, Maria Mancini, Sarah McDonald, Laurence McEvoy, John McLauchlan, Soeren Metelmann, Nahida S Miah, Joanna Middleton, Joyce Mitchell, Shona C Moore, Ellen G Murphy, Rebekah PenriceRandal, Jack Pilgrim, Tessa Prince, Will Reynolds, P Matthew Ridley, Debby Sales, Victoria E Shaw, Rebecca K Shears, Benjamin Small, Krishanthi S Subramaniam, Agnieska Szemiel, Aislynn Taggart, Jolanta Tanianis-Hughes, Jordan Thomas, Erwan Trochu, Libby van Tonder, Eve Wilcock, J Eunice Zhang; local principal investigators: Kayode Adeniji, Daniel Agranoff, Ken Agwuh, Dhiraj Ail, Ana Alegria, Brian Angus, Abdul Ashish, Dougal Atkinson, Shahedal Bari, Gavin Barlow, Stella Barnass, Nicholas Barrett, Christopher Bassford, David Baxter, Michael Beadsworth, Jolanta Bernatoniene, John Berridge, Nicola Best, Pieter Bothma, David Brealey, Robin Brittain-Long, Naomi Bulteel, Tom Burden, Andrew Burtenshaw, Vikki Caruth, David Chadwick, Duncan Chambler, Nigel Chee, Jenny Child, Srikanth Chukkambotla, Tom Clark, Paul Collini, Catherine Cosgrove, Jason Cupitt, Maria-Teresa Cutino-Moguel, Paul Dark, Chris Dawson, Samir Dervisevic, Phil Donnison, Sam Douthwaite, Ingrid DuRand, Ahilanadan Dushianthan, Tristan Dyer, Cariad Evans, Chi Eziefula, Chrisopher Fegan, Adam Finn, Duncan Fullerton, Sanjeev Garg, Sanjeev Garg, Atul Garg, Jo Godden, Arthur Goldsmith, Clive Graham, Elaine Hardy, Stuart Hartshorn, Daniel Harvey, Peter Havalda, Daniel B Hawcutt, Maria Hobrok, Luke Hodgson, Anita Holme, Anil Hormis, Michael Jacobs, Susan Jain, Paul Jennings, Agilan Kaliappan, Vidya Kasipandian, Stephen Kegg, Michael Kelsey, Jason Kendall, Caroline Kerrison, lan Kerslake, Oliver Koch, Gouri Koduri, George Koshy, Shondipon Laha, Susan Larkin, Tamas Leiner, Patrick Lillie, James Limb, Vanessa Linnett, Jeff Little, Michael MacMahon, Emily MacNaughton, Ravish Mankregod, Huw Masson, Elijah Matovu, Katherine McCullough, Ruth McEwen, Manjula Meda, Gary Mills, Jane Minton, Mariyam Mirfenderesky, Kavya Mohandas, Quen Mok, James Moon, Elinoor Moore, Patrick Morgan, Craig Morris, Katherine Mortimore, Samuel Moses, Mbiye Mpenge, Rohinton Mulla, Michael Murphy, Megan Nagel, Thapas Nagarajan, Mark Nelson, Igor Otahal, Mark Pais, Selva Panchatsharam, Hassan Paraiso, Brij Patel, Justin Pepperell, Mark Peters, Mandeep Phull, Stefania Pintus, Jagtur Singh Pooni, Frank Post, David Price, Rachel Prout, Nikolas Rae, Henrik Reschreiter, Tim Reynolds, Neil Richardson, Mark Roberts, Devender Roberts, Alistair Rose, Guy Rousseau, Brendan Ryan, Taranprit Saluja, Aarti Shah, Prad Shanmuga, Anil Sharma, Anna Shawcross, Jeremy Sizer, Richard Smith, Catherine Snelson, Nick Spittle, Nikki Staines, Tom Stambach, Richard Stewart, Pradeep Subudhi, Tamas Szakmany, Kate Tatham, Jo Thomas, Chris Thompson, Robert Thompson, Ascanio Tridente, Darell Tupper-Carey, Mary Twagira, Andrew Ustianowski, Nick Vallotton, Lisa Vincent-Smith, Shico Visuvanathan, Alan Vuylsteke, Sam Waddy, Rachel Wake, Andrew Walden, Ingeborg Welters, Tony Whitehouse, Paul Whittaker, Ashley Whittington, Meme Wijesinghe, Martin Williams, Lawrence Wilson, Sarah Wilson, Stephen Winchester, Martin Wiselka, Adam Wolverson, Daniel G Wooton, Andrew Workman, Bryan Yates, Peter Young.

\section{Role of the funder/sponsor}

The funder/sponsor had no role in the design and conduct of the study; collection, management, analysis, and interpretation of the data; preparation, 
review, or approval of the manuscript; and decision to submit the manuscript for publication.

\section{Authors' contributions}

TY, FZ and KK conceptualised the idea; TY, FZ, NI, CR, CLG, CAL, YC, AR, MJD helped design the work; ABD, PJMO, JKB, MGS acquired the data; TY analysed the data; TY, FZ, NI, CR, CLG, CAL, YC, AR, MJD helped with interpretation of the data; TY drafted the work; TY, FZ, NI, CR, CLG, CAL, YC, AR, MJD, ABD, PJMO, JKB, MGS and KK (all authors) approved the submitted version and agree to be personally accountable for their own contributions related to the work.

\section{Funding}

This work was supported by the NIHR Leicester BRC, NIHR ARC-EM and a grant from the UKRI-DHSC COVID-19 Rapid Response Rolling Call (MR/ V020536/1), the National Institute for Health Research (NIHR; award CO-CIN01), the Medical Research Council (MRC; grant MC_PC_19059), and by the NIHR Health Protection Research Unit (HPRU) in Emerging and Zoonotic Infections at University of Liverpool.

\section{Availability of data and materials}

The data that support the findings of this study are available from International Severe Acute Respiratory and emerging Infections Consortium (ISARIC) but restrictions apply to the availability of these data, which were used under license for the current study, and so are not publicly available. Data are however available from the authors upon reasonable request and with permission of ISARIC4C. If someone wishes to request the data from this study, please contact the ISARIC4C consortium (https://isaric4c.net/) for access enquiries.

\section{Declarations}

\section{Ethics approval and consent to participate}

Ethical approval for data collection and analysis by ISARIC4C was given by the South Central-Oxford C Research Ethics Committee in England (reference 13/SC/0149), and by the Scotland A Research Ethics Committee (reference 20/SS/0028). The ISARIC WHO CCP-UK study was registered at https:// www.isrctn.com/ISRCTN66726260 and designated an Urgent Public Health Research Study by NIHR. The methods used to collect the human data for this study were completed in accordance with all the relevant guidelines and regulations. Informed consent or proxy consent was collected from all human participants for their data to be included in this study. Further information on the methods to collect data, informed consent and study can be found within the study protocol, which can be found at https://isaric4c.net/.

\section{Consent for publication}

Not applicable.

\section{Competing interests}

KK is supported by the National Institute for Health Research (NIHR) Applied Research Collaboration East Midlands (ARC EM) and TY by the NIHR Leicester Biomedical Research Centre (BRC). KK is Director for the University of Leicester Centre for BME Health, Trustee of the South Asian Health Foundation, national NIHR ARC lead for Ethnicity and Diversity and a member of Independent SAGE and Chair of the SAGE subgroup on ethnicity and COVID-19. MGS is a member of HMG SAGE COVID-19. MGS reports grants from DHSC NIHR UK, grants from MRC UK, grants from HPRU in Emerging and Zoonotic Infections, University of Liverpool, during the conduct of the study, other from Integrum Scientific LLC, Greensboro, NC, USA, outside the submitted work. Other authors declare no conflicts of interests.

\section{Author details}

${ }^{1}$ Diabetes Research Centre, Leicester General Hospital, University of Leicester, Leicester LE5 4PW, UK. ${ }^{2}$ National Institute for Health Research (NIHR) Leicester Biomedical Research Centre (BRC), Leicester General Hospital, Leicester LE5 4PW, UK. ${ }^{3}$ Leicester Real World Evidence Unit, Diabetes Research Centre, University of Leicester, Leicester, UK. ${ }^{4}$ Clinical Trial Service Unit and Epidemiological Studies Unit (CTSU), Nuffield Department of Population Health, University of Oxford, Oxford, UK. ${ }^{5}$ Medical Research Council Epidemiology Unit, University of Cambridge, Cambridge, UK. ${ }^{6}$ Centre for Medical Informatics, Usher Institute, University of Edinburgh, Edinburgh,
UK. ${ }^{7}$ Intensive Care Unit, Royal Infirmary Edinburgh, Edinburgh, UK. ${ }^{8}$ National Heart and Lung Institute, Imperial College London, London, UK. ${ }^{9}$ Roslin Institute, University of Edinburgh, Edinburgh, UK. ${ }^{10} \mathrm{NIHR}$ Health Protection Research Unit in Emerging and Zoonotic Infections and Institute of Translational Medicine, Faculty of Health and Life Sciences, University of Liverpool, Liverpool, UK. ${ }^{11}$ Respiratory Medicine, Alder Hey Children's Hospital, Institute in The Park, University of Liverpool, Alder Hey Children's Hospital, L12 2AP, Liverpool, UK. ${ }^{12}$ NIHR Applied Research Collaboration East Midlands (ARC-EM), Leicester General Hospital, Leicester, UK.

\section{Received: 16 February 2021 Accepted: 19 July 2021}

Published online: 31 July 2021

\section{References}

1. Kambhampati SBS, Vaishya R, Vaish A. Unprecedented surge in publications related to COVID-19 in the first three months of pandemic: a bibliometric analytic report. J Clin Orthop Trauma. 2020;11(Suppl 3):S304-6. https://doi. org/10.1016/j.jcot.2020.04.030.

2. Williamson EJ, Walker AJ, Bhaskaran K, Bacon S, Bates C, Morton CE, et al. Factors associated with COVID-19-related death using OpenSAFELY. Nature. 2020;584(7821):430-6. https://doi.org/10.1038/s41586-020-2521-4.

3. Docherty AB, Harrison EM, Green CA, Hardwick HE, Pius R, Norman L, et al. Features of 20133 UK patients in hospital with covid-19 using the ISARIC WHO Clinical Characterisation Protocol: prospective observational cohort study. BMJ. 2020;369:m1985.

4. Seidu S, Gillies C, Zaccardi F, Kunutsor SK, Hartmann-Boyce J, Yates T,Singh AK, Davies MJ, Khunti K. The impact of obesity on severe disease and mortality in people with SARS-CoV-2: A systematic review and metaanalysis. Endocrinol Diabetes Metab. 2020;4(1):e00176. https://doi.org/10.1 002/edm2.176. Epub ahead of print.

5. Singh AK, Gillies CL, Singh R, Singh A, Chudasama Y, Coles B, Seidu S, Zaccardi F, Davies MJ, Khunti K. The Prevalence of Comorbidities and Their Association with Mortality in Patients with COVID-19: A Systematic Review and Meta-Analysis. Available at SSRN 3576854. 2020.

6. Ssentongo P, Ssentongo AE, Heilbrunn ES, Ba DM, Chinchilli VM. Association of cardiovascular disease and 10 other pre-existing comorbidities with COVID-19 mortality: a systematic review and meta-analysis. PLoS One. 2020; 15(8):e0238215. https://doi.org/10.1371/journal.pone.0238215.

7. Mahumud RA, Kamara JK, Renzaho AM. The epidemiological burden of and overall distribution of chronic comorbidities in coronavirus disease-2019 among 202,005 infected patients: evidence from a systematic review and meta-analysis. Infection. 2020;19:1-21.

8. Barron E, Bakhai C, Kar P, Weaver A, Bradley D, Ismail H, et al. Associations of type 1 and type 2 diabetes with COVID-19-related mortality in England: a whole-population study. Lancet Diabetes Endocrinol. 2020;8(10):813-22. https://doi.org/10.1016/S2213-8587(20)30272-2.

9. Islam N, Khunti K, Dambha-Miller H, Kawachi I, Marmot M. COVID-19 mortality: a complex interplay of sex, gender and ethnicity. Eur J Pub Health. 2020;30(5):847-8. https://doi.org/10.1093/eurpub/ckaa150.

10. Lighter J, Phillips M, Hochman S, Sterling S, Johnson D, Francois F, et al. Obesity in patients younger than 60 years is a risk factor for Covid-19 hospital admission. Clin Infect Dis. 2020;71(15):896-7. https://doi.org/10.1 093/cid/ciaa415.

11. Kass DA, Duggal P, Cingolani O. Obesity could shift severe COVID-19 disease to younger ages. Lancet (London, England). 2020;395(10236):1544-5.

12. Tartof SY, Qian L, Hong V, Wei R, Nadjafi RF, Fischer H, et al. Obesity and mortality among patients diagnosed with COVID-19: results from an integrated health care organization. Ann Intern Med. 2020. https://doi.org/1 0.7326/M20-3742.

13. Fresán U, Guevara M, Elía F, Albéniz E, Burgui C, Castilla J, et al. Independent role of morbid obesity as a risk factor for COVID-19 hospitalization: a Spanish population-based cohort study. Obesity. 2020. https://doi.org/10.1 002/oby.23029.

14. Hendren NS, de Lemos JA, Ayers C, Das SR, Rao A, Carter S, et al. Association of Body Mass Index and Age With Morbidity and Mortality in Patients Hospitalized With COVID-19: Results From the American Heart Association COVID-19 Cardiovascular Disease Registry. Circulation. 2020. https://doi.org/10.1161/circulationaha.120.051936.

15. Czernichow S, Beeker N, Rives-Lange C, Guerot E, Diehl JL, Katsahian S, et al. AP-HP/Universities/Inserm COVID-19 research collaboration and AP-HP Covid CDR Initiative. Obesity doubles mortality in patients hospitalized for 
SARS-CoV-2 in Paris hospitals, France: a cohort study on 5795 patients. Obesity. 2020. https://doi.org/10.1002/oby.23014.

16. Dunning JW, Merson L, Rohde GG, Gao Z, Semple MG, Tran D, et al. Open source clinical science for emerging infections. Lancet Infect Dis. 2014;14(1): 8-9. https://doi.org/10.1016/S1473-3099(13)70327-X.

17. Depart of Health and Social Care, UK. Priority groups for coronavirus (COVID-19) vaccination: advice from the JCVI. 2020. https://www.gov.uk/ government/publications/priority-groups-for-coronavirus-covid-19-vaccina tion-advice-from-the-jcvi-2-december-2020/priority-groups-for-coronaviruscovid-19-vaccination-advice-from-the-jcvi-2-december-2020 Accessed 17 Dec 2020

18. Conolly A, Davies B. Health survey for England 2017_-adult and child overweight and obesity. Leeds: NHS Digital, NHS; 2018. https://digital.nhs. uk/data-and-information/publications/statistical/health-survey-for-england/2 017 assessed 12/11/2020

19. Greenhalgh T, Knight M, Buxton M, Husain L. Management of post-acute covid-19 in primary care. BMJ. 2020;370.

\section{Publisher's Note}

Springer Nature remains neutral with regard to jurisdictional claims in published maps and institutional affiliations.

Ready to submit your research? Choose BMC and benefit from:

- fast, convenient online submission

- thorough peer review by experienced researchers in your field

- rapid publication on acceptance

- support for research data, including large and complex data types

- gold Open Access which fosters wider collaboration and increased citations

- maximum visibility for your research: over $100 \mathrm{M}$ website views per year

At $\mathrm{BMC}$, research is always in progress.

Learn more biomedcentral.com/submissions 